\title{
PET of Glucose Metabolism and Cellular Proliferation in Prostate Cancer
}

\author{
Hossein Jadvar \\ Division of Nuclear Medicine, Department of Radiology, Keck School of Medicine of USC, University of Southern California, \\ Los Angeles, California
}

Imaging of the Warburg effect, which is the principal but not the sole cause for enhanced glucose metabolism in tumors, with PET and ${ }^{18} \mathrm{~F}-\mathrm{FDG}$ has become the mainstay for the imaging evaluation of several cancers. Despite the seemingly prevalent notion that ${ }^{18} \mathrm{~F}-\mathrm{FDG}$ PET may not be useful in prostate cancer, relatively limited evidence suggests that this imaging modality can be useful for the evaluation of the extent of metastatic disease and the assessment of the therapy response and prognosis in men with castration-resistant prostate cancer. Incidental high focal ${ }^{18} \mathrm{~F}-\mathrm{FDG}$ uptake in the prostate gland, although generally rare, may also indicate occult prostate cancer that may need to be further scrutinized. In general, ${ }^{18} \mathrm{~F}-\mathrm{FDG}$ PET is not useful for initial staging and is of limited utility in the clinical setting of biochemical failure after prior definitive therapy for primary cancer. Although more experience is needed, it appears that the imaging of cellular proliferation with PET and $3^{\prime}$-deoxy- $3^{\prime}-{ }^{18} \mathrm{~F}$-fluorothymidine or $2^{\prime}-{ }^{18} \mathrm{~F}$-fluoro-5-methyl-1- $\beta$-D-arabinofuranosyluracil may also allow for targeted biopsy and localization for focal therapy of aggressive prostate tumors as well as assessment of the therapy response to various standard and novel treatment regimens in patients with metastatic disease.

Key Words: PET/CT; PET; prostate cancer; ${ }^{18} \mathrm{~F}-\mathrm{FDG}$; ${ }^{18} \mathrm{~F}-\mathrm{FLT}$; ${ }^{18}$ F-FMAU

J Nucl Med 2016; 57:25S-29S

DOI: 10.2967/jnumed.115.170704

\section{I} terest in the potential role of PET with several radiotracers targeted to the underlying complex biology of prostate cancer has been increasing. The Warburg effect is a hallmark of cancer and can be reliably interrogated with PET and ${ }^{18} \mathrm{~F}$-FDG. In fact, ${ }^{18} \mathrm{~F}-\mathrm{FDG}$ PET has now become the mainstay for the imaging evaluation of several cancers. Another important biologic feature in cancer is cellular proliferation. The imaging of cellular proliferation can allow for tumor characterization and early objective assessment of the response to therapy. This article summarizes experience with the utility and limitations of PET for the imaging examination of glucose metabolism and cellular proliferation in prostate cancer.

\footnotetext{
Received Feb. 29, 2016; revision accepted Mar. 22, 2016.

For correspondence or reprints contact: Hossein Jadvar, Division of Nuclear Medicine, Department of Radiology, Keck School of Medicine of USC, University of Southern California, 2250 Alcazar St., CSC 102, Los Angeles, CA 90033.

E-mail: jadvar@med.usc.edu.

COPYRIGHT (c) 2016 by the Society of Nuclear Medicine and Molecular Imaging, Inc.
}

\section{GLUCOSE METABOLISM}

It has often been stated that ${ }^{18} \mathrm{~F}$-FDG PET is not useful in prostate cancer. However, this belief seems to have arisen from some early studies in which ${ }^{18}$ F-FDG PET was interrogated in the setting of primary tumor diagnosis or staging of the disease, for which the overall results were unsatisfactory $(1-3)$. The utility of ${ }^{18} \mathrm{~F}-\mathrm{FDG}$ PET appears to depend on the phase of the disease; therefore, it may be quite relevant in one phase of the disease but limited in another phase $(4,5)$.

\section{Incidental High Prostatic ${ }^{18}$ F-FDG Uptake in Primary Cancer Detection}

Reesink et al. assessed the clinical relevance of incidental prostatic lesions with ${ }^{18}$ F-FDG PET and whether the findings should prompt additional evaluations (6). That investigation involved 108 consecutive men who had bladder cancer and underwent radical cystoprostatectomy. Incidental prostatic uptake was noted in $40 \%$ of the cohort; overall, occult prostate cancers were found in $23 \%$ of the surgical specimens. The positive and negative predictive values for findings labeled as suspect or indeterminate for prostate cancer were $29 \%$ and $79 \%$, respectively. However, the authors' final conclusions were that overall incidental prostatic uptake on ${ }^{18} \mathrm{~F}-\mathrm{FDG}$ $\mathrm{PET} / \mathrm{CT}$ had a low positive predictive value for prostate cancer and that the Gleason score did not correlate with the $\mathrm{SUV}_{\max }$ or serum prostate-specific antigen (PSA).

In another study, involving 6,128 male patients who had undergone ${ }^{18} \mathrm{~F}$-FDG PET scans, incidental prostatic ${ }^{18} \mathrm{~F}$-FDG uptake was noted in $1.3 \%$ of the patients (7). There was no significant correlation between $\mathrm{SUV}_{\max }$ or serum PSA levels and whether the lesions were benign or malignant. Brown et al. reported that focal incidental prostatic uptake with an $\mathrm{SUV}_{\max }$ of greater than 6 should be further evaluated with multiparametric MRI (8). A recent systematic review and metaanalysis of 47,925 men in 6 studies reported a pooled prevalence of $1.8 \%$ ( $95 \%$ confidence interval [CI], $1.3 \%-2.3 \%$ ) for incidental high ${ }^{18} \mathrm{~F}-\mathrm{FDG}$ uptake in the prostate gland (9). The pooled risks of malignancy in patients who were further evaluated or underwent biopsy (444 patients with incidental prostatic uptake underwent further evaluation and 121 patients underwent biopsy) were 17\% (95\% CI, 12\%-23\%) and $62 \%(95 \%$ CI, 54\%-71\%), respectively. Kang et al. suggested that incidental prostatic uptake on ${ }^{18} \mathrm{~F}$-FDG PET scans should not be ignored and that further investigation, such as PSA determination or additional imaging, should be undertaken; they made this suggestion despite the realization that the level of ${ }^{18} \mathrm{~F}-\mathrm{FDG}$ accumulation can overlap in normal prostate, benign prostatic hyperplasia, and prostate cancer tissues, which often coexist (10).

Kwon et al. reported that, of 47,109 men who underwent ${ }^{18}$ F-FDG PET in a 10-y period between 2004 and 2014, 1,335 (2.83\%) showed 
incidental prostatic ${ }^{18} \mathrm{~F}$-FDG uptake and 99 of these men underwent prostate biopsy (11). Prostate cancer occurred in $3.8 \%$ of men with serum PSA levels of less than $2.5 \mathrm{ng} / \mathrm{mL}$ and in $59.7 \%$ of men with serum PSA levels of greater than or equal to $2.5 \mathrm{ng} / \mathrm{mL}$. Multivariable analysis showed that focal lesions (odds ratio, 5.50; $P=0.038$ ), age (odds ratio, 1.06; $P=0.031$ ), and serum PSA levels (odds ratio, $1.28 ; P=0.001$ ) were independent predictors of prostate cancer diagnosis. The authors concluded that patients with high ${ }^{18} \mathrm{~F}$-FDG uptake in the prostate should be further evaluated by the measurement of serum PSA and that those with high serum PSA levels should be considered for prostate biopsy. In another Japanese investigation, an incidental prostatic ${ }^{18} \mathrm{~F}-\mathrm{FDG}$ uptake of $2 \%$ in 3,236 cases was reported (12). In the evaluable 49 cases, $16 \%$ had prostate cancer, whereas $84 \%$ were benign.

\section{Initial Staging}

There are few data on the use of ${ }^{18} \mathrm{~F}-\mathrm{FDG}$ PET/CT for the initial staging of prostate cancer, given the general low avidity of ${ }^{18} \mathrm{~F}-$ FDG for primary prostate cancer. Liu reported on a retrospective study of 9 men (mean serum PSA level, $291 \mathrm{ng} / \mathrm{mL}$; SD, $363 \mathrm{ng} / \mathrm{mL}$; range, $6.1-980 \mathrm{ng} / \mathrm{mL}$ ) who underwent ${ }^{18} \mathrm{~F}-\mathrm{FDG}$ PET/CT at the time of initial staging of known primary prostate cancer (13). The standard of reference for the PET observations was biopsy, regional diagnostic $\mathrm{CT}$, or whole-body bone scan. Although the sensitivity of ${ }^{18} \mathrm{~F}-\mathrm{FDG}$ PET/CT for identifying primary cancer was only $33 \%$, metastatic lymph nodes or bone lesions were also detected in 6 of the 9 patients. Liu concluded that, in general, ${ }^{18}$ F-FDG PET/CT may not be useful for the detection of primary cancer but may be useful for initial staging in certain subgroups of patients with high serum PSA levels.

Beauregard et al. performed ${ }^{18} \mathrm{~F}-\mathrm{FDG}$ PET/CT for the staging workup of 44 patients with known Gleason sum scores of greater than or equal to 8 (i.e., aggressive tumors) (14). Foci suggesting high ${ }^{18} \mathrm{~F}$-FDG uptake were found in the prostate gland, lymph nodes, and bone in $44 \%, 13 \%$, and $6 \%$ of the patients, respectively. The absence or presence of intraprostatic ${ }^{18} \mathrm{~F}-\mathrm{FDG}$ uptake was associated with a median cancer-free survival probability of $70.2 \%$ or $26.9 \%$ ( $P=0.0097)$, respectively.

In the early analysis of the National Oncologic PET Registry data in the United States, involving 2,042 scans, for the initial staging of prostate cancer (the most common cancer type in the initial staging subgroup), ${ }^{18} \mathrm{~F}-\mathrm{FDG}$ PET/CT had an impact on clinical management in $32 \%(95 \% \mathrm{CI}, 30.0 \%-34.1 \%)$ of the patients (15).

\section{Biochemical Recurrence}

Localization of disease in patients with biochemical recurrence is essential, as it directs appropriate management, which may include salvage therapy with surgery or radiation for local recurrence, systemic therapy for metastatic disease, or both. The American Urologic Association defines biochemical recurrence in postprostatectomy patients as an initial serum PSA level of $0.2 \mathrm{ng} / \mathrm{mL}$ or higher, with a second, confirmatory level of greater than $0.2 \mathrm{ng} / \mathrm{mL}$ (16). The American Society for Therapeutic Radiology and Oncology consensus definition for biochemical failure after primary external-beam radiotherapy is an increase of $2 \mathrm{ng} / \mathrm{mL}$ or more above the nadir PSA level, regardless of hormonal therapy (17). Nonstandard imaging studies should only be considered when the results of standard imaging ( ${ }^{99 \mathrm{~m}} \mathrm{Tc}$-based bone scintigraphy or contrast-enhanced abdomen and pelvis CT) are negative or equivocal. Multiparametric MRI is also typically used to scrutinize the prostate bed.

In a study of ${ }^{18}$ F-FDG PET, a sensitivity of $75 \%$ and a specificity of $100 \%$ for the detection of pelvic lymph node metastases were reported; validation was based on histopathologic examination of the surgically harvested nodes (18). Jadvar et al. reported the findings of a prospective investigation on the potential utility of ${ }^{18} \mathrm{~F}-\mathrm{FDG}$ PET/CT and ${ }^{18} \mathrm{~F}-\mathrm{NaF}$ PET/CT for the detection of occult metastases in 37 men with PSA relapse (range, 0.5-40.2 $\mathrm{ng} / \mathrm{mL}$ ) and strictly negative results on standard imaging studies (19). The ${ }^{18}$ F-FDG PET/CT detection rate was only $8.1 \%$ in the setting of biochemical recurrence. In another recent investigation, involving 28 patients with PSA relapse after definitive primary therapy $(82.1 \%$ radical prostatectomy and $17.9 \%$ external-beam radiation therapy), the sensitivity and specificity of ${ }^{18} \mathrm{~F}-\mathrm{FDG}$ PET/ CT were $61.6 \%$ and $75 \%$, respectively (20). Schöder et al. reported a positive detection rate of $31 \%$ in this clinical setting (21). In another comparative study of ${ }^{18} \mathrm{~F}-\mathrm{FDG}$ and ${ }^{11} \mathrm{C}$-choline, the sensitivities of ${ }^{11} \mathrm{C}$-choline and ${ }^{18} \mathrm{~F}$-FDG were $60.6 \%$ and $31 \%$, respectively (22). The sensitivities increased for both radiotracers, to $80 \%$ and $40 \%$, respectively, when the serum PSA levels were greater than $1.9 \mathrm{ng} / \mathrm{mL}$. On the basis of current experience, it appears that, in general, ${ }^{18} \mathrm{~F}-\mathrm{FDG}$ PET has limited utility in this clinical setting.

\section{Response Assessment in Metastatic Disease}

Prostate cancer is a remarkably heterogeneous disease; therefore, a personalized approach to tailored treatment is most desired. Such an approach demands surrogate imaging markers that can portray the disease activity accurately before, during, and after treatment as well as dependence on specific response criteria that are used in data analysis, such as RECIST 1.0, RECIST 1.1, or PERCIST $1.0(23,24)$. Tumor ${ }^{18} \mathrm{~F}$-FDG uptake generally decreases with successful treatment (androgen deprivation or chemotherapy), although imaging findings may be discordant with those of other manifestations of disease, including changes in the levels of serum PSA or circulating tumor cells (25).

Simoncic et al. compared dynamic ${ }^{18} \mathrm{~F}-\mathrm{NaF}$ and ${ }^{18} \mathrm{~F}-\mathrm{FDG}$ PET/CT for assessment of the response to zibotentan in men with bone metastases from prostate cancer (26). Late (2-wk break after 4 wk of therapy, i.e., wk 6) ${ }^{18} \mathrm{~F}-\mathrm{NaF}$ and ${ }^{18} \mathrm{~F}-\mathrm{FDG}$ uptake responses were correlated, but earlier uptake responses ( $4 \mathrm{wk}$ of therapy) were unrelated, suggesting that ${ }^{18} \mathrm{~F}-\mathrm{NaF}$ uptake and ${ }^{18} \mathrm{~F}$-FDG uptake in the setting of response assessment may be spatially disjointed and that these radiotracers may provide complementary information. Other studies have shown that ${ }^{18} \mathrm{~F}$-FDG uptake in metastatic lesions declines with successful androgen deprivation therapy or chemotherapy (Fig. 1) (27,28). Although these preliminary studies are encouraging, there is clearly a need for additional experience in this clinical scenario.

\section{Assessment of Prognosis}

Recently, there has been increasing emphasis on the prognostic utility of various imaging studies in cancer, in terms of accuracy for the prediction of an outcome of interest, which can help with clinical management decisions and with assessment of the comparative effectiveness of various conventional and emerging treatment strategies. In the clinical setting of prostate cancer, these outcome measures may include, but are not limited to, time to biochemical recurrence (time to PSA progression), time to first metastasis, time to symptomatic progression, time to initiation of cytotoxic chemotherapy, time to radiographic progression, time to castration resistance state, progression-free survival, metastasis-free survival, disease-specific survival, and overall survival (29).

In an investigation of 42 men with primary prostate cancer, Oyama et al. showed that patients with higher primary tumor 


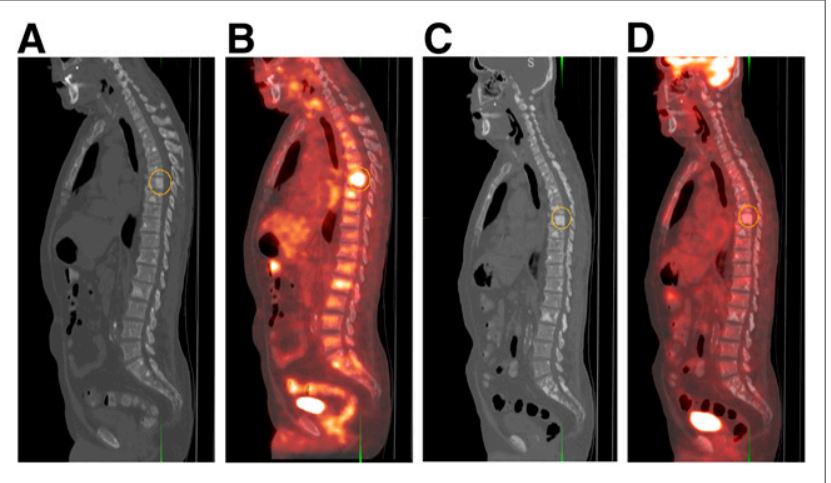

FIGURE 1. 69-y-old man with castration-resistant metastatic prostate cancer before $(A$ and $B)$ and after ( $C$ and $D)$ treatment with docetaxel. Note general decline in metabolic activity in bone lesions (index thoracic spine lesion SUV $V_{\text {max }}$, denoted by circular region of interest, declined from 5.0 to 2.1), compatible with favorable response to chemotherapy and concordant with decline in serum PSA level from $98 \mathrm{ng} / \mathrm{mL}$ before therapy to $21 \mathrm{ng} / \mathrm{mL}$ after therapy. (A and C) Sagittal CT at bone window level. (B and D) Fused ${ }^{18}$ F-FDG PET/CT.

uptake had a significantly poorer prognosis than did patients with tumors that showed lower ${ }^{18} \mathrm{~F}-\mathrm{FDG}$ uptake (30). Meirelles et al. compared the prognostic values of bone scans and ${ }^{18} \mathrm{~F}-\mathrm{FDG}$ PET in a prospective imaging trial of 43 men with metastatic castrationresistant prostate cancer (31). Overall survival correlated inversely with the $\mathrm{SUV}_{\max }$ of the osseous lesions, with median survival times of 14.4 mo for $\mathrm{SUV}_{\max }$ of greater than 6.10 and 32.8 mo for $\mathrm{SUV}_{\max }$ of less than or equal to $6.10(P=0.002)$. Although a calculated bone scan index was also prognostic (14.7 mo and 28.2 mo for bone scan indices of $>1.27$ and $<1.27$, respectively; $P=0.004$ ), in the multivariate analysis, only $\mathrm{SUV}_{\max }$ was an independent factor for predicting survival.

Jadvar et al. reported on a prospective study of 87 men who had metastatic castration-resistant prostate cancer, underwent ${ }^{18} \mathrm{~F}-\mathrm{FDG}$ $\mathrm{PET} / \mathrm{CT}$, and were then monitored for overall survival (32). In the multivariate analysis after adjustment for prognostic clinical confounders (age, serum PSA level, serum alkaline phosphatase level, use of pain medication, prior chemotherapy, and Gleason score at initial diagnosis), the sum of the $\mathrm{SUV}_{\max }$ for up to 25 metabolically active lesions (lymph node, bone, and soft-tissue metastases) was

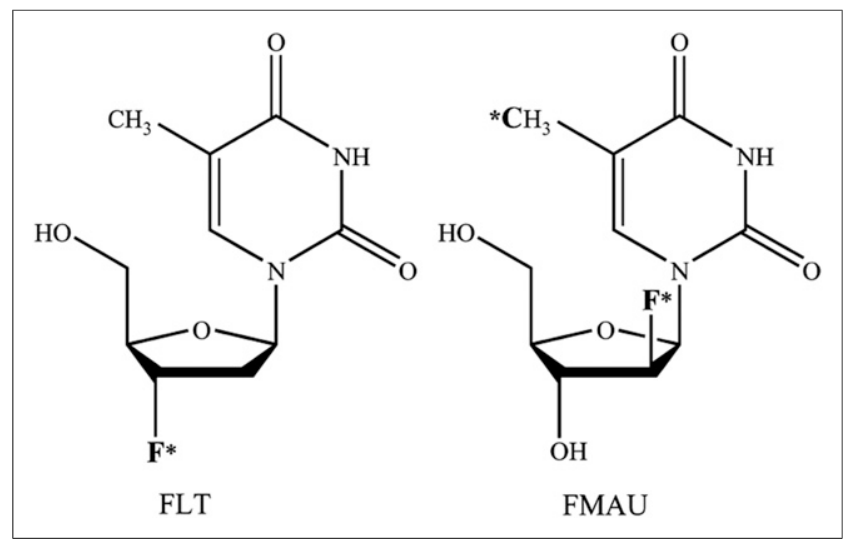

FIGURE 2. Chemical structures of ${ }^{18} \mathrm{~F}-\mathrm{FLT}$ and ${ }^{18} \mathrm{~F}-\mathrm{FMAU} . \mathrm{F}^{*}$ denotes position of ${ }^{18} \mathrm{~F}$ for ${ }^{18} \mathrm{~F}-\mathrm{FLT}$ and ${ }^{18} \mathrm{~F}$-FMAU; ${ }^{*} \mathrm{C}$ denotes position of ${ }^{11} \mathrm{C}$ for ${ }^{11} \mathrm{C}$-FMAU. (Reprinted with permission of (37).)

statistically significant, with a hazard ratio of 1.01 (95\% CI, 1.001$1.020 ; P=0.053$ ), for predicting overall survival. Specifically, the moving hazard of death in relation to the sum of the $\mathrm{SUV}_{\max }$, interpreted as the chance of death per person per month, showed a marked upward shift of the curve (i.e., increased chance of death) for a sum of the $\mathrm{SUV}_{\max }$ of greater than 20 .

In another retrospective investigation, the association of CT patterns and glycolytic activity of prostate cancer bone metastases with overall survival was investigated in 38 patients (33). The number of lesions on CT or ${ }^{18} \mathrm{~F}$-FDG PET, but not the intensity of ${ }^{18} \mathrm{~F}$-FDG uptake, was associated with overall survival.

Aside from differences in methodology between the study of Jadvar et al. and the study of Vargas et al., the central hypothesis remains the same: that both the number of lesions and the intensity or aggressiveness of the "worst" lesion will be independent prognostic variables (34).

\section{CELLULAR PROLIFERATION}

The imaging of cellular proliferation provides valuable information about the rate of tumor growth, which can be important in tumor characterization (e.g., indolent vs. aggressive), and early assessment of the response to therapy (35). PET in conjunction with radiotracers that track the thymidine salvage pathway of DNA synthesis has been studied for the noninvasive imaging-based assessment of cellular proliferation in cancer (36-38). Experience with 2 radiotracers that have been used in preclinical and pilot clinical studies of prostate cancer, $3^{\prime}$-deoxy- $3^{\prime}-{ }^{18}$ F-fluorothymidine $\left({ }^{18} \mathrm{~F}-\mathrm{FLT}\right)$ and $2{ }^{\prime}-{ }^{18} \mathrm{~F}$-fluoro5 -methyl-1- $\beta$-D-arabinofuranosyluracil ( $\left.{ }^{18} \mathrm{~F}-\mathrm{FMAU}\right)$, is briefly highlighted here (Fig. 2).

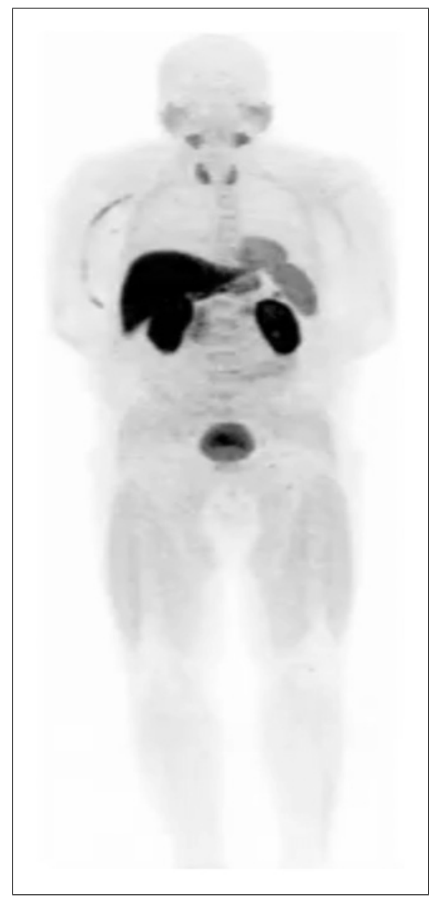

FIGURE 3. Normal biodistribution of ${ }^{18} \mathrm{~F}-\mathrm{FMAU}$. Note relatively high tracer uptake in liver and renal cortex; moderate uptake in salivary glands, thyroid, heart, spleen, and urinary bladder; and relatively low uptake in bone marrow.

\section{${ }^{18}$ F-FLT}

${ }^{18} \mathrm{~F}-\mathrm{FLT}$ is the most studied cellular proliferation PET tracer. It is phosphorylated by thymidine kinase 1 , is retained in proliferating cells without DNA incorporation, and can be described with a 3-compartment model $(39,40)$. Normal biodistribution is characterized by relatively high uptake in the liver and bone marrow, with the urinary bladder receiving the highest dose through renal excretion (41). Other than data from a few preclinical animal studies, few data on the potential utility of ${ }^{18}$ F-FLT in human prostate cancer are available, perhaps because of the high physiologic localization of ${ }^{18} \mathrm{~F}$-FLT in normal bone marrow-the most common site for prostate tumor metastases. Nevertheless, a preclinical micro-PET study demonstrated a significant decline in ${ }^{18}$ F-FLT uptake after docetaxel treatment in $22 \mathrm{Rv} 1$ hormonerefractory prostate tumors 


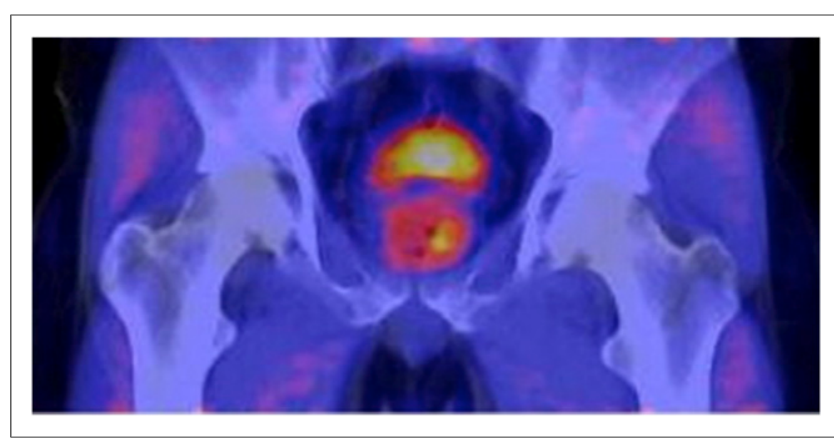

FIGURE 4. 61-y-old man who had elevated serum PSA level $(10.5 \mathrm{ng} / \mathrm{mL})$ and had undergone standard transrectal ultrasound biopsy, with negative results. Axial PET/CT with ${ }^{18} \mathrm{~F}-\mathrm{FMAU}$ demonstrated focally increased tracer uptake in left base of prostate gland. PET/multiparametric MRI-directed biopsy revealed atypical small acinar proliferation suggestive of early malignancy.

implanted in athymic mice (42). The conclusion was that ${ }^{18} \mathrm{~F}$-FLT might be a useful tracer for the early assessment of anticancer therapy with docetaxel in patients with castration-resistant prostate cancer.

\section{F-FMAU}

${ }^{18} \mathrm{~F}-\mathrm{FMAU}$ is phosphorylated by thymidine kinase and incorporated into DNA (43). Tehrani et al. showed that this thymidine analog was preferentially phosphorylated by mitochondrial thymidine kinase 2 rather than cytosolic thymidine kinase 1 (44). The fact that ${ }^{18} \mathrm{~F}$-FMAU shows little accumulation in bone renders it an ideal PET radiotracer in prostate cancer (Fig. 3) (45). Jadvar et al. showed that there may be an association between androgen signaling and thymidine metabolism and that ${ }^{18}$ F-FMAU PET may be useful in prostate tumor characterization (46). A possible explanation may be the androgen control of mitochondrial function, which may include thymidine kinase 2 enzymatic activity (47). A pilot observational study of ${ }^{18} \mathrm{~F}$-FMAU PET in 3 men with prostate cancer confirmed the tumor retention of ${ }^{18} \mathrm{~F}-\mathrm{FMAU}$ in local prostate recurrence and in metastatic lesions with barely visible activity in the urinary bladder and normal bone (48). Moreover, on average, $95 \%$ of the blood activity cleared within $10 \mathrm{~min}$ after ${ }^{18} \mathrm{~F}-\mathrm{FMAU}$ administration, and about $70 \%$ of the activity in the urine was intact ${ }^{18} \mathrm{~F}-\mathrm{FMAU}$ at $60 \mathrm{~min}$ after injection. Jadvar et al. also recently initiated a pilot study to assess the potential utility of ${ }^{18} \mathrm{~F}-\mathrm{FMAU}$ in image-targeted biopsy with software-based fusion of PET, transrectal ultrasound, and multiparametric MRI of the prostate gland (Fig. 4) (49). This hybrid imaging methodology may allow for improved localization and characterization of tumors for targeted biopsy and focal therapy (50).

\section{CONCLUSION}

Incidental high focal ${ }^{18} \mathrm{~F}$-FDG uptake in the prostate gland is rare but may identify previously unknown prostate tumors. ${ }^{18} \mathrm{~F}-\mathrm{FDG}$ PET is generally not useful for staging known disease and has limited value in patients with biochemical recurrence. Castrationresistant metastatic disease is often metabolically active, and limited evidence currently suggests that ${ }^{18}$ F-FDG PET may be useful for assessment of treatment response and prognosis. Imaging of cellular proliferation with ${ }^{18}$ F-FMAU may allow for the localization of aggressive primary tumors, which may then be amenable to focal therapy of localized prostate cancer.

\section{DISCLOSURE}

Hossein Jadvar was supported in part by National Institutes of Health grants R01-CA111613, R21-CA142426, R21-EB017568, and P30-CA014089. No other potential conflict of interest relevant to this article was reported.

\section{REFERENCES}

1. Salminen E, Hogg A, Binns D, Frydenberg M, Hicks R. Investigations with FDG PET scanning in prostate cancer show limited value for clinical practice. Acta Oncol. 2002;41:425-429.

2. Liu IJ, Zafar MB, Lai YH, et al. Fluorodeoxyglucose positron emission tomography studies in diagnosis and staging of clinically organ-confined prostate cancer. Urology. 2001;57:108-111.

3. Hofer C, Laubenbacher C, Block T, et al. Fluorine-18-fluorodeoxyglucose positron emission tomography is useless for detection of local recurrence after radical prostatectomy. Eur Urol. 1999;36:31-35.

4. Jadvar H. Molecular imaging of prostate cancer with [F-18] fluorodeoxyglucose PET. Nat Rev Urol. 2009;6:317-323.

5. Jadvar H. Imaging evaluation of prostate cancer with ${ }^{18} \mathrm{~F}$-fluorodeoxyglucose PET/CT: utility and limitations. Eur J Nucl Med Mol Imaging. 2013;40(suppl 1):S5-S10.

6. Reesink DJ, Fransen van de Putte EE, Vegt E, et al. Clinical relevance of incidental prostatic lesions on FDG-positron emission tomography/computerized tomography: should patients receive further evaluation? J Urol. November 17, 2015 [Epub ahead of print].

7. Sahin E, Elboga U, Kalender E, Basibuyuk M, Demir HD, Celen YZ. Clinical significance of incidental FDG uptake in the prostate gland detected by PET/CT. Int J Clin Exp Med. 2015;8:10577-10585.

8. Brown AM, Lindenberg ML, Sankineni S, et al. Does focal incidental ${ }^{18} \mathrm{~F}-\mathrm{FDG}$ uptake in the prostate gland have significance? Abdom Imaging. 2015;40:32223229.

9. Bertagna F, Sadeghi R, Giovanella L, et al. Incidental uptake of ${ }^{18}$ F-fluorodeoxyglucose in the prostate gland: systematic review and meta-analysis on prevalence and risk of malignancy. Nuklearmedizin. 2014;53:249-258.

10. Kang PM, Seo WI, Lee SS, et al. Incidental abnormal FDG uptake in the prostate on 18-fluoro-2-deoxyglucose positron emission tomography-computed tomography scans. Asian Pac J Cancer Prev. 2014;15:8699-8703.

11. Kwon T, Jeong IG, You D, et al. Prevalence and clinical significance of incidental ${ }^{18} \mathrm{~F}$-fluorodeoxyglucose uptake in prostate. Korean J Urol. 2015;56: 288-294.

12. Seino $\mathrm{H}$, Ono $\mathrm{S}$, Miura $\mathrm{H}$, et al. Incidental prostate ${ }^{18} \mathrm{~F}-\mathrm{FDG}$ uptake without calcification indicates possibility of prostate cancer. Oncol Rep. 2014;31:15171522 .

13. Liu Y. Diagnostic role of fluorodeoxyglucose positron emission tomographycomputed tomography in prostate cancer. Oncol Lett. 2014;7:2013-2018.

14. Beauregard JM, Blouin AC, Fradet V, et al. FDG-PET/CT for pre-operative staging and prognostic stratification of patients with high-grade prostate cancer at biopsy. Cancer Imaging. 2015;15:2.

15. Hillner BE, Siegel BA, Shields AF, et al. Relationship between cancer type and impact of PET and PET/CT on intended management: findings of the National Oncologic PET Registry. J Nucl Med. 2008;49:1928-1935.

16. Cookson MS, Aus G, Burnett AL, et al. Variation in the definition of biochemical recurrence in patients treated for localized prostate cancer: the American Urological Association Prostate Guidelines for Localized Prostate Cancer Update Panel report and recommendations for a standard in the reporting of surgical outcomes. J Urol. 2007;177:540-545.

17. Roach M III, Hanks G, Thames H Jr, et al. Defining biochemical failure following radiotherapy with or without hormonal therapy in men with clinically localized prostate cancer: recommendation of the RTOG-ASTRO Phoenix Consensus Conference. Int J Radiat Oncol Biol Phys. 2006;65: 965-974.

18. Chang $\mathrm{CH}$, Wu HU, Tsai JJ, et al. Detecting metastatic pelvic lymph nodes by ${ }^{18} \mathrm{~F}$-2-deoxyglucose positron emission tomography in patients with prostate specific antigen relapse after treatment for localized prostate cancer. Urol Int. 2003;70:311-315.

19. Jadvar H, Desai B, Ji L, et al. Prospective evaluation of ${ }^{18} \mathrm{FNaF}$ and ${ }^{18} \mathrm{~F}-\mathrm{FDG}$ $\mathrm{PET} / \mathrm{CT}$ in detection of occult metastatic disease in biochemical recurrence of prostate cancer. Clin Nucl Med. 2012;37:637-643.

20. Öztürk H, Karapolat I. ${ }^{18}$ F-fluorodeoxyglucose PET/CT for detection of disease in patients with prostate-specific antigen relapse following radical treatment of a local-stage prostate cancer. Oncol Lett. 2016;11:316-322. 
21. Schöder H, Herrmann K, Gönen M, et al. 2-[18F]fluoro-2-deoxyglucose positron emission tomography for the detection of disease in patients with prostate-specific antigen relapse after radical prostatectomy. Clin Cancer Res. 2005;11:4761-4769.

22. Richter JA, Rodríguez M, Rioja J, et al. Dual tracer ${ }^{11} \mathrm{C}$-choline and FDG-PET in the diagnosis of biochemical prostate cancer relapse after radical treatment. Mol Imaging Biol. 2010;12:210-217.

23. Jadvar H. Prostate cancer: PET with ${ }^{18} \mathrm{~F}-\mathrm{FDG},{ }^{18} \mathrm{~F}$ - or ${ }^{11} \mathrm{C}$-acetate, and ${ }^{18} \mathrm{~F}$ - or ${ }^{11}$ C-choline. J Nucl Med. 2011;52:81-89.

24. Jadvar H, Desai B, Ji L, et al. RECIST 1.0, PERCIST 1.0 and PSA treatment response criteria in metastatic castrate-resistant prostate cancer [abstract]. In: Radiological Society of North America 99th Scientific Assembly and Annual Meeting; December 1-6, 2013; Chicago, IL.

25. Jadvar H, Desai B, Ji L, et al. Comparison of RECIST 1.0, PERCIST 1.0 and PCWG2 treatment response criteria in metastatic castrate-sensitive prostate cancer [abstract]. In: Society of Nuclear Medicine and Molecular Imaging Annual Meeting; June 6-10, 2015; Baltimore, MD.

26. Simoncic U, Perlman S, Liu G, Staab MJ, Straus JE, Jeraj R. Comparison of NaF and FDG PET/CT for assessment of treatment response in castrate-resistant prostate cancers with osseous metastases. Clin Genitourin Cancer. 2015;13:e7-e17.

27. Oyama N, Akino H, Suzuki Y, et al. FDG PET for evaluating the change of glucose metabolism in prostate cancer after androgen ablation. Nucl Med Commun. 2001;22:963-969.

28. Jadvar H, Desai B, Quinn D, et al. Treatment response assessment of metastatic prostate cancer with FDG PET/CT [abstract]. J Nucl Med. 2011;52(suppl 1):1908.

29. Jadvar H. Prognostic utility of PET in prostate cancer. PET Clin. 2015;10:255-263.

30. Oyama N, Akino H, Suzuki Y, et al. Prognostic value of 2-deoxy-2-[F-18]fluoroD-glucose positron emission tomography imaging for patients with prostate cancer. Mol Imaging Biol. 2002;4:99-104.

31. Meirelles GS, Schoder H, Ravizzini GC, et al. Prognostic value of baseline $\left[{ }^{18} \mathrm{~F}\right]$ fluorodeoxyglucose positron emission tomography and ${ }^{99 \mathrm{~m}} \mathrm{Tc}-\mathrm{MDP}$ bone scan in progressing metastatic prostate cancer. Clin Cancer Res. 2010;16:6093-6099.

32. Jadvar H, Desai B, Ji L, et al. Baseline ${ }^{18} \mathrm{~F}$-FDG PET/CT parameters as imaging biomarkers of overall survival in castrate-resistant metastatic prostate cancer. J Nucl Med. 2013;54:1195-1201.

33. Vargas HA, Wassberg C, Fox JJ, et al. Bone metastases in castration-resistant prostate cancer: associations between morphologic CT patterns, glycolytic activity, and androgen receptor expression on PET and overall survival. Radiology. 2014;271:220-229.

34. Jadvar H, Groshen SG, Quinn DI. Association of overall survival with glycolytic activity of castrate-resistant prostate cancer metastases. Radiology. 2015;274:624-625.
35. Mankoff DA, Shields AF, Krohn KA. PET imaging of cellular proliferation. Radiol Clin North Am. 2005;43:153-167.

36. Nimmagadda S, Shields AF. The role of DNA synthesis imaging in cancer in the era of targeted therapeutics. Cancer Metastasis Rev. 2008;27:575-587.

37. Bading JR, Shields AF. Imaging of cell proliferation: status and prospects. J Nucl Med. 2008;49(suppl 2):64S-80S.

38. Tehrani OS, Shields AF. PET imaging of proliferation with pyrimidines. J Nucl Med. 2013;54:903-912.

39. Shields AF, Briston DA, Chandupatla $S$, et al. A simplified analysis of $\left[{ }^{18} \mathrm{~F}\right] 3^{\prime}-$ deoxy-3'-fluorothymidine metabolism and retention. Eur J Nucl Med Mol Imaging. 2005;32:1269-1275.

40. Shields AF, Grierson JR, Dohmen BM, et al. Imaging proliferation in vivo with [F-18]FLT and positron emission tomography. Nat Med. 1998;4:1334-1336.

41. Vesselle H, Grierson J, Peterson LM, Muzi M, Mankoff DA, Krohn KA. ${ }^{18}$ Ffluorothymidine radiation dosimetry in human PET imaging studies. J Nucl Med. 2003;44:1482-1488.

42. Oyama N, Hasegawa Y, Kiyono Y, et al. Early response assessment in prostate carcinoma by ${ }^{18} \mathrm{~F}$-fluorothymidine following anticancer therapy with docetaxel using preclinical tumor models. Eur J Nucl Med Mol Imaging. 2011;38: 81-89.

43. Sun H, Mangner TJ, Collins JM, Muzik O, Douglas K, Shields AF. Imaging DNA synthesis in vivo with ${ }^{18}$ F-FMAU and PET. J Nucl Med. 2005;46:292296.

44. Tehrani OS, Douglas KA, Lawhorn-Crews JM, Shields AF. Tracking cellular stress with labeled FMAU reflects changes in mitochondrial TK2. Eur J Nucl Med Mol Imaging. 2008;35:1480-1488.

45. Shields AF. Positron emission tomography measurement of tumor metabolism and growth: its expanding role in oncology. Mol Imaging Biol. 2006;8:141-150.

46. Jadvar H, Yap LP, Park R, et al. $\left[{ }^{18} \mathrm{~F}\right]-2^{\prime}$-fluoro-5-methyl-1-beta-D-arabinofuranosyluracil ( $\left.{ }^{18} \mathrm{~F}-\mathrm{FMAU}\right)$ in prostate cancer: initial preclinical observations. $\mathrm{Mol}$ Imaging. 2012;11:426-432.

47. Doeg KA, Polomski LL, Doeg LH. Androgen control of mitochondrial and nuclear DNA synthesis in male sex accessory tissue of castrate rats. Endocrinology. 1972;90:1633-1638.

48. Sun H, Sloan A, Mangner TJ, et al. Imaging DNA synthesis with $\left[{ }^{18} \mathrm{~F}\right] \mathrm{FMAU}$ and positron emission tomography in patients with cancer. Eur J Nucl Med Mol Imaging. 2005;32:15-22.

49. Jadvar H, Chen K, Ukimura O. Targeted prostate gland biopsy with combined TRUS, mpMRI and ${ }^{18}$ F-FMAU PET-CT. Clin Nucl Med. 2015;40:e426-e428.

50. Jadvar $\mathrm{H}$. Imaging cellular proliferation in prostate cancer with positron emission tomography. Asia Ocean J Nucl Med Biol. 2015;3:72-76. 